\title{
Noninvasive Tests of Fibrosis: To Get the Right Answers, We Need to Ask the Right Questions
}

\author{
Amreen Dinani ${ }^{1}$, Jordan J. Feld ${ }^{1^{*}}$ \\ ${ }^{1}$ Toronto Western Hospital Liver Centre, University Health Network, University of Toronto, Toronto, Canada
}

\section{A R T I C L E I N F O}

Article Type:

Letter to Editor

Article history:

Received: 14 Apr 2011

Revised: 01 May 2011

Accepted: 11 May 2011

Keywords:

Fibrosis

Aspartate aminotransferases

Fatty liver

\section{Dear Editor,}

We read with interest the work by Yimaz et al. entitled 'Noninvasive assessment of liver fibrosis with the aspartate transaminase to platelet ratio index (APRI): Usefulness in patients with chronic liver disease' in th journal of Hepatity Monthly (1). This study adds to the growing body of literature showing the utility of the APRI as a noninvasive method to assess liver fibrosis. This retrospective study included 455 patients with different liver diseases: 207 patients with chronic hepatitis B (CHB), 108 with chronic hepatitis C (CHC), and 140 patients with nonalcoholic fatty liver disease (NAFLD). APRI scores were calculated and compared to liver biopsy findings in all patients. ROC curves were created to assess the accuracy of the APRI as a predictor of the absence or presence of fibrosis (Fo vs. F1-4). The authors concluded that APRI was significantly associated with fibrosis scores in patients with CHC and NAFLD, but not in those with CHB. This is a well-designed study with adequate sample size that addresses an important clinical question. We agree with the authors that the

\footnotetext{
* Corresponding author at: Jordan J. Feld, Toronto Western Hospital Liver Centre, University Health Network, University of Toronto, Toronto, Canada. Tel:+1-4165818595, +1-4166036230. Fax:+1-4166035472.

E-mail: Jordan.Feld@uhn.on.ca

DOI:10.5812/kowsar.1735143X.706

Copyright @ 2011, BRCGL, Published by Kowsar M.P.Co. All rights reserved.
}

- Please cite this paper as:

Dinani A, Feld JJ. Noninvasive Tests of Fibrosis: To Get the Right Answers, We Need to Ask the Right Questions. Hepat Mon. 2011; 11(8):668-9.

[DOI:10.5812/kowsar.1735143X.706]

๑ 2011 Kowsar M.P.Co. All rights reserved.

APRI is a good tool for the assessment of liver fibrosis, with some notable caveats. The authors chose to use the ROC curves to assess the utility of the APRI as a predictor of absence or presence of fibrosis. Alternatively, the ROC curves could have been created with various APRI cutoffs to predict the absence or presence of cirrhosis. Using cirrhosis rather than any fibrosis may be more clinically relevant because the presence of cirrhosis determines the urgency of treatment, serves as a prognostic marker for a therapeutic outcomes and establishes the need to monitor of complications. Perhaps more important is that, the performance of APRI may actually be much better for determining if cirrhosis has an effect than for identifying the presence of any fibrosis. The two variables that constitute APRI are AST and platelet count, both of witch are markers of advanced fibrosis and cirrhosis $(2,3)$. AST is a mitochondrial enzyme that increases disproportionately more than ALT and thus increases the AST/ALT ratio in cirrhosis with progressive liver injury (4). Furthermore, the platelet count declines as liver disease progresses due to splenic sequestration from portal hypertension and eventually from thrombopoietin deficiency with very impaired liver synthetic function. Both of these parameters may be entirely normal in patients with early-stage fibrosis, and therefore it is not surprising that APRI was useful but far from foolproof in excluding fibrosis. 
Interestingly, the authors found that the APRI was less predictive of fibrosis in patients with CHB compared to the other liver diseases. Elloumi et al. (5) and Sebastiani et al. (6) have also showed similar results. In all these studies, areas under the ROC curves for fibrosis in CHB were not impressive (0.54, 0.62 and 0.64 respectively). This probably reflects the dynamic nature of CHB. Because the APRI includes the AST levels, it may be greatly influenced by a flare of disease, a common occurrence in CHB. In contrast in CHC and NAFLD, the degree of inflammation and hence the AST tend to be relatively stable over time.

Another point for consideration is the way witch we define the thresholds using the ROC curves. The authors chose the points that maximized both specificity and sensitivity, as is the usual practice. However, in this particular instance, it might be more useful to choose the cutoff to maximize sensitivity, even with a resultant loss in specificity. One would want the highest sensitivity so that all patients with any degree of fibrosis are being identified to ensure that progressive liver disease is not missed. Using the thresholds from the ROC curves in this study, the sensitivities were modest at best: $72.2 \%$,
60.0\% and 55\% for CHC, NAFLD, and CHB, respectively. If the analyses were redone to identify advanced fibrosis, maximizing specificity would become more important.

Finally, we were somewhat confused by a comment made by the authors that only, 'CHC and NAFLD have been associated with low platelet counts.' In general, all chronic liver diseases with progressive liver fibrosis and even noncirrhotic portal hypertension are associated with thrombocytopenia. Notably, Karasu et al. (7) found a negative correlation between platelet counts and stage of fibrosis in univariate and multivariate analyses for both $\mathrm{CHC}$ and $\mathrm{CHB}$ patients.

In conclusion, the authors have collected a great deal of data to answer an important clinical question. With the data at hand, it would be interesting to examine whether APRI performed better as a marker of advanced liver disease, and it would also be very interesting to compare APRI to other noninvasive tests such as FIB-4 and FORNS, witch could easily be calculated with the existing data. Noninvasive markers are certainly an important tool, but at least for now, liver pathologists need not worry about their job security.

\title{
Author's Reply; Noninvasive Tests of Fibrosis: To Get the Right Answers, We Need to Ask the Right Questions
}

\author{
Yusuf Yilmaz ${ }^{1,2 *}$, Ramazan Kurt ${ }^{1,2}$ \\ ${ }^{1}$ Department of Gastroenterology, Marmara University, School of Medicine, Istanbul, Turkey \\ ${ }^{2}$ Institute of Gastroenterology, Marmara University, Istanbul, Turkey
}

\section{A R T I C L E I N F O}

Article Type:

Letter to Editor

Article history:

Received: 09 May 2011

Revised: 12 May 2011

Accepted: 17 May 2011

\section{Keywords:}

Fatty liver

Chronic hepatitis B

Liver fibrosis

\section{Dear Editor,}

We thank Drs Dinani and Feld for their interest in our article (1). Their letter raises interesting questions regar-

\footnotetext{
* Corresponding author at: Yusuf Yilmaz, Department of Gastroenterology, Marmara University, School of Medicine, Istanbul, Turkey. Tel: +905334403995, Fax:+90-2166886681.

E-mail:yusufyilmaz@uludag.edu.tr DOI:10.5812/kowsar.1735143X.706

Copyright $\odot 2011$, BRCGL, Published by Kowsar M.P.Co. All rights reserved.
}

Please cite this paper as:

Yilmaz Y, Kurt R. Author's Reply; Noninvasive Tests of Fibrosis: To Get the Right Answers, We Need to Ask the Right Questions. Hepat Mon. 2011; 11(8):669-70.

[DOI: 10.5812/kowsar.1735143X.706]

๑ 2011 Kowsar M.P.Co. All rights reserved.

ding the usefulness of the APRI index in the context of chronic liver diseases. First, the authors argued that the APRI index would be more useful to detect advanced liver fibrosis or cirrhosis rather than early stage fibrosis. Some of the earliest clinical studies on this potential value of the APRI index have already shown the value of this noninvasive parameter in predicting cirrhosis in patients with chronic liver diseases of various causes $(8,9)$. In our study, we did not try to replicate these findings; ra- 
ther, we focused on the question as to whether the APRI index could generally predict the presence of hepatic fibrosis. Although our results have shown that the sensitivity for the detection of fibrosis was optimum, there is an urgent need for sensitive biomarkers of early-stage fibrosis, when therapeutic interventions can be more effective than in the cirrhosis stage. Second, Drs. Dinani and Feld suggested that the low value of the APRI index for predicting fibrosis in chronic HBV infection could be due to disease flares associated with fluctuations in liver transaminases. We absolutely agree with this potential explanation. Clearly, this possibility needs to be adequately tested in future studies that should consider the fluctuating nature of chronic HBV infection. Third, the authors suggested performing a ROC curve analyses to identify the cut-off to maximize sensitivity for the detection of fibrosis, even with a resulting loss in specificity. This is a valuable suggestion, but we believe that future studies on the hepatology community in the next few years should concentrate on the improving the detection of fibrosis by adding additional noninvasive biomarkers to the currently available simple noninvasive indexes (such as the APRI). This goal may help to attain a clinically reasonable trade-off between sensitivity and specificity. Finally, Drs. Dinani and Feld were puzzled by our statement that "CHC and NAFLD have been associated with low platelet counts". We agree with the fact that chronic HBV infection can be similarly accompanied by thrombocytopenia. However, there is evidence that platelet count may be generally normal in patients with chronic HBV infection, with thrombocytopenia being common only in the presence of advanced cirrhosis or fibrosis (10).

\section{References}

1. Yilmaz Y, Yonal O, Kurt R, Bayrak M, Aktas B, Ozdogan O. Noninvasive assessment of liver fibrosis with the aspartate transaminase to platelet ratio index (APRI): Usefulness in patients with chronic liver disease. Hepat Mon. 2011;11(2):103-6.

2. Khokhar N. Serum aminotransferase levels and platelet count as predictive factor of fibrosis and cirrhosis in patients with chronic hepatitis C infection. J Pak Med Assoc. 2003;53(3):101-4.

3. Pohl A, Behling C, Oliver D, Kilani M, Monson P, Hassanein T. Serum aminotransferase levels and platelet counts as predictors of degree of fibrosis in chronic hepatitis C virus infection. Am J Gastroenterol. 2001;96(11):3142-6.

4. Assy N, Minuk GY. Serum aspartate but not alanine aminotransferase levels help to predict the histological features of chronic hepatitis $C$ viral infections in adults. Am J Gastroenterol. 2000;95(6):1545-50.

5. Elloumi H, Hefaiedh R, Khayatt O, Chedly A, Zouari B, BelHadj N, et al. APRI score: Is it Useful for Assessment of Fibrosis in Chronic Hepatitis B? Arab J Gastroenterol ;. 2007;8(4):136-7.

6. Sebastiani G, Vario A, Guido M, Alberti A. Sequential algorithms combining non-invasive markers and biopsy for the assessment of liver fibrosis in chronic hepatitis B. World J Gastroenterol. 2007;13(4):525-

7. Karasu Z, Tekin F, Ersoz G, Gunsar F, Batur Y, Ilter T, et al. Liver fibrosis is associated with decreased peripheral platelet count in patients with chronic hepatitis B and C. Dig Dis Sci. 2007;52(6):1535-9.

8. Forestier J, Dumortier J, Guillaud O, Ecochard M, Roman S, Boillot $\mathrm{O}$, et al. Noninvasive diagnosis and prognosis of liver cirrhosis: a comparison of biological scores, elastometry, and metabolic liver function tests. Eur J Gastroenterol Hepatol. 2010;22(5):532-40.

9. Fujii H, Enomoto M, Fukushima W, Ohfuji S, Mori M, Kobayashi S, et al. Noninvasive laboratory tests proposed for predicting cirrhosis in patients with chronic hepatitis $C$ are also useful in patients with non-alcoholic steatohepatitis. J Gastroenterol. 2009;44(6):608-14.

10. Nwokediuko SC, Ibegbulam O. Quantitative Platelet Abnormalities in Patients With Hepatitis B Virus-Related Liver Disease. Gastroenterol Res. 2010;2(6):344-9.

\section{Financial Disclosures}

None declared. 